\title{
Inhibition of autophagy enhances cisplatin-induced apoptosis in the MG63 human osteosarcoma cell line
}

\author{
ZHICAI ZHANG, ZENGWU SHAO, LIMING XIONG and SHUHUA YANG \\ Department of Orthopedics, Union Hospital, Tongji Medical College, Huazhong University of Science and Technology, \\ Wuhan, Hubei 430022, P.R. China
}

Received December 24, 2014; Accepted August 17, 2015

DOI: 10.3892/ol.2015.3692

\begin{abstract}
Autophagy is a significant catabolic process that allows the renewal of intracellular organelles, through which cells are able to maintain homeostasis. In addition, autophagy may be associated with the carcinogenesis of osteosarcoma (OS). Cisplatin (CDDP) is an alkylating agent that is commonly used as an anticancer therapy. However, the pathways underlying the effects of CDDP remain to be elucidated. The present study demonstrated that 3-methyladenine (3-MA), an inhibitor of autophagy, was able to increase the proliferation inhibition ratios of MG63 human OS cells when used in combination with CDDP. Furthermore, MG63 cells produced significantly more microtubule-associated protein light chain 3II (LC3II), a widely used marker for monitoring autophagy, following CDDP treatment. Treatment with 3-MA was observed to inhibit these changes. Similarly, MG63 cells co-treated with 3-MA and CDDP demonstrated increased sensitivity to CDDP-induced apoptosis, compared with those exposed to CDDP alone. The present study revealed variation in the expression of LC3II and caspase-3 activity following treatment with certain drugs. The results of the present study suggest that CDDP may be capable of inducing apoptosis and autophagy, and that autophagy may be able to inhibit apoptosis in MG63 cells. Therefore, downregulation of autophagy may increase the chemotherapeutic sensitivity of MG63 cells to CDDP.
\end{abstract}

\section{Introduction}

Osteosarcoma (OS) is the most commonly observed primary bone tumor in children (1). It is an aggressive malignant tumor, and $>80 \%$ of patients treated with surgery alone exhibit distant metastases (2). Standard therapy is typically multimodal, consisting of neoadjuvant chemotherapy and subsequent

Correspondence to: Dr Zengwu Shao, Department of Orthopedics, Union Hospital, Tongji Medical College, Huazhong University of Science and Technology, 1277 Jiefang Avenue, Wuhan, Hubei 430022, P.R. China

E-mail: szwxhpro@126.com

Key words: osteosarcoma, autophagy, apoptosis, 3-methyladenine, cisplatin amputation or limb-sparing reconstructive surgeries, in combination with adjuvant chemotherapy (3). CDDP, a common antitumor drug, has been found to be effective against cancer cells derived from solid tumors, such as osteosarcoma, hepatoma and thymoma. In clinical treatment, CDDP is a common choice for osteosarcoma chemotherapy (4).

Advances in molecular technologies have facilitated an increased understanding of the mechanisms underlying carcinogenesis, primarily focusing on the recognized model of multistage carcinogenesis with underlying progressive genetic changes, which induce malignant transformation (5). Recently, the mechanisms underlying autophagy have gained increasing attention.

Autophagy describes the bulk degradation of proteins and organelles, and is an essential process for effective cellular maintenance, viability, differentiation and mammalian development (6). In mammals, autophagy has been observed in numerous tissues, and has been demonstrated to possess significant associations with neurodegenerative diseases, cardiomyopathies, tumors and apoptosis, as well as bacterial and viral infections (7). It has been proposed that autophagy may be capable of protecting certain cancer cells from anticancer therapies by blocking apoptotic pathways, whereas other cancer cells are observed to undergo autophagic cell death following anticancer therapy (8). The opening of the mitochondrial permeability transition pore induces autophagy and apoptosis, and a small quantity of mitochondria spontaneously depolarize and induce autophagy (9). In this way, autophagy eliminates dysfunctional mitochondria, so that cells are protected from apoptosis.

Whilst several signaling pathways are implicated in autophagic cell death, the PI3K-AKT-mTOR signaling pathway appears to play a pivotal role. 3-MA, an inhibitor of class III PI3K, has been used to suppress autophagy (10). Microtubule-associated protein light chain 3II (LC3II) is increasingly being used to monitor levels of autophagy; the expression levels of LC3II have been observed to correlate with the number of autophagosomes, and therefore this is potentially a useful method (11).

In the present study, MG63 OS cell proliferation inhibition ratios were evaluated following the application of various drugs. In particular, the present study focused on apoptosis, the formation of autophagosomes under various conditions and the expression of characteristic autophagy genes, including LC3II and the apoptosis-activated protein caspase-3. 


\section{Materials and methods}

Cell culture and experimental design. The MG63 human OS cell line was cultured in RPMI-1640 supplemented with $10 \%$ heat-inactivated fetal bovine serum (FBS; both from HyClone, Logan, UT, USA), $1 \%$ penicillin $(50 \mathrm{U} / \mathrm{ml}) / \mathrm{strep}$ tomycin $(50 \mu \mathrm{g} / \mathrm{ml})$ (Gibco Life Technologies, Carlsbad, CA, USA) and $5 \mu \mathrm{g} / \mathrm{ml}$ Plasmocin ${ }^{\mathrm{TM}}$ prophylactic (InvivoGen, San Diego, CA, USA) in a humidified atmosphere with $5 \% \mathrm{CO}_{2}$, in a water-jacketed incubator at $37^{\circ} \mathrm{C}$. The MG63 cell line was obtained from the China Center for Typical Culture Collection (Wuhan University, Wuhan, Hubei, China).

CDDP and 3-MA were purchased from Sigma-Aldrich (St. Louis, MO, USA). MG63 cells were divided into four groups: Control group, cisplatin (CDDP) group, 3-methyladenine (3-MA) group and CDDP + 3-MA group. Control group cells were treated with phosphate-buffered saline (PBS; SigmaAldrich). The CDDP group was exposed to CDDP $(10 \mu \mathrm{g} / \mathrm{ml})$, the 3-MA group was treated with 3-MA $(6 \mathrm{mM})$ and the CDDP + 3-MA group was treated with CDDP $(10 \mu \mathrm{g} / \mathrm{ml})$ and 3 -MA $(6 \mathrm{mM})$. Cells were exposed to the drugs for $24 \mathrm{~h}$.

MTT assay. MG63 cells were cultured until mid-log phase in order to obtain a stock cell suspension that contained $1 \times 10^{8} \mathrm{cells} / 1$. The stock cell suspension (100 $\mu$ l) was subsequently added to a 96-well plate. Following $24 \mathrm{~h}$ of culture, cells were treated with the drugs, and subsequently incubated at $37^{\circ} \mathrm{C}$ in $5 \% \mathrm{CO}_{2}$ for $24 \mathrm{~h}$. Following incubation, $20 \mu \mathrm{l}$ MTT stock solution $(5 \mathrm{mg} / \mathrm{ml}$ in PBS; Amresco LLC, Solon, OH, USA) was added to each well. Cells were subsequently incubated at $37^{\circ} \mathrm{C}$ for $4 \mathrm{~h}$, then centrifuged at $1,000 \mathrm{x} \mathrm{g}$ for $10 \mathrm{~min}$ at $37^{\circ} \mathrm{C}$. Supernatant was discarded, $150 \mu 1$ dimethyl sulfoxide (Sigma-Aldrich) was added and the plate was agitated for 10 min in the dark. Finally, the optical density (OD) was detected using a microplate reader ( $\mu$ Quant $^{\mathrm{TM}}$; Bio-Tek Instruments, Inc., Winooski, VT, USA) at a wavelength of $490 \mathrm{~nm}$. The following formula was utilized in order to calculate cell proliferation inhibition ratios: Inhibition ratio $=\left[1-\left(\mathrm{OD}_{\text {experimental samples }} / \mathrm{OD}_{\text {contro }}\right)\right] \times 100 \%$.

Laser scanning confocal microscopy. Cells in the four groups were incubated in culture media with various supplements, as indicated. Following $24 \mathrm{~h}$ of incubation, cells were plated on coverslips and fixed in $4 \%$ formaldehyde (Sigma-Aldrich) in PBS for 20 min at $4^{\circ} \mathrm{C}$, rinsed in PBS and exposed to an incubation buffer (5\% FBS in PBS) for an additional $20 \mathrm{~min}$ at room temperature. Cells were subsequently treated with 1:200 diluted polyclonal rabbit anti-human IgG antibody against LC3II (\#sc28266; Santa Cruz Biotechnology, Inc., Dallas, TX, USA) for $12 \mathrm{~h}$ in a humidifier at $4^{\circ} \mathrm{C}$. Following rinsing in the incubation buffer (twice for $5 \mathrm{~min}$ ), the specimens were incubated with 1:100-diluted goat anti-rabbit IgG-fluorescein isothiocyanate (FITC) conjugate (\#F-2765; Invitrogen Life Technologies, Carlsbad, CA, USA) and propidium iodide (PI; Sigma-Aldrich) for $1 \mathrm{~h}$ at room temperature, and subsequently rinsed with PBS. Fluorescence was detected using a laser confocal microscope (TCS SP2; Leica Microsystems GmbH, Wetzlar, Germany).

Flow cytometry. Following treatment for $24 \mathrm{~h}$ with various drugs, cells were collected by centrifugation at $1500 \mathrm{x}$ g for $10 \mathrm{~min}$ at $4^{\circ} \mathrm{C}$, fixed in $4 \%$ formaldehyde, rinsed with PBS and

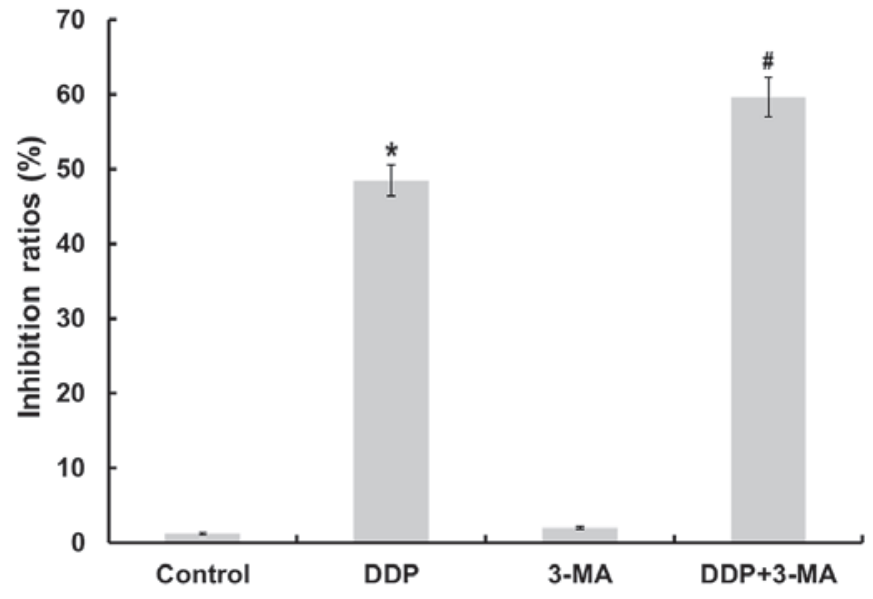

Figure 1. 3-MA enhances CDDP-induced inhibition of cell proliferation. Cell proliferation inhibition ratios of MG63 cells treated with various drugs. MG63 cells were treated with CDDP $(10 \mu \mathrm{g} / \mathrm{ml})$, autophagy inhibitor 3-MA (6 mM), or co-treated with CDDP and 3-MA, and evaluated using an MTT assay. Results are presented as the mean \pm standard error for combined data from five separate experiments. CDDP significantly increased the cell proliferation inhibition ratio of MG63 cells compared with that of untreated control cells $\left({ }^{*} \mathrm{P}<0.05\right)$. CDDP + 3-MA significantly increased the cell proliferation inhibition ratio of MG63 cells compared with that of cells treated with CDDP alone $(\mathrm{P}<0.05)$. There was no significant difference between the control and 3-MA groups ( $\mathrm{P}>0.05)$. CDDP, cisplatin; 3-MA, 3-methyladenine.

exposed to an incubation buffer at $4^{\circ} \mathrm{C}$. Cells were treated with 1:200 diluted polyclonal rabbit anti-human antibody to LC3II for $12 \mathrm{~h}$ at $4^{\circ} \mathrm{C}$. Following rinsing with the incubation buffer, specimens were exposed to 1:100 diluted goat anti-rabbit IgG-FITC conjugate for $1 \mathrm{~h}$ at room temperature and subsequently rinsed with PBS.

Apoptosis was quantified by combined staining with Annexin V and PI using an Annexin V-FITC Apoptosis Detection kit (MBL International Co., Woburn, MA, USA). Briefly, $24 \mathrm{~h}$ subsequent to treatment with various drugs, cells were collected by centrifugation at $1500 \mathrm{xg}$ for $10 \mathrm{~min}$ at $4^{\circ} \mathrm{C}$, and dissolved in $500 \mu \mathrm{l} 1 \mathrm{X}$ binding buffer. Following the addition of $10 \mu \mathrm{l}$ Annexin V-FITC solution and $5 \mu \mathrm{l}$ PI solution, cells were incubated for $15 \mathrm{~min}$ at room temperature in the dark.

Following incubation, cells were analyzed using a flow cytometer (FACSort; BD Biosciences, Franklin Lakes, NJ, USA) equipped with an argon laser and filter configuration for FITC/PI dye combination. Light scattering and fluorescence signals were subjected to linear and logarithmic amplifications, respectively. At least 10,000 events were acquired and analyzed using Cell Quest software version 5.1 (BD Biosciences). All the experiments were performed at least three times for each condition. The data were plotted on a logarithmic scale.

Western blot analysis. Cytosolic fraction proteins were separately collected using an Apo Alert Cell Fractionation kit (Clontech Laboratories, Inc., Mountainview, CA, USA). MG63 cells were trypsinized (Sigma-Aldrich), collected, resuspended in ice-cold wash buffer and then centrifuged at $700 \mathrm{xg}$ for $5 \mathrm{~min}$ at $4^{\circ} \mathrm{C}$. Following removal of the supernatant, cells were resuspended in $200 \mu 1$ ice-cold fractionation buffer mix and placed on ice for $10 \mathrm{~min}$. The homogenates were centrifuged at $10,000 \mathrm{xg}$ for $10 \mathrm{~min}$ at $4^{\circ} \mathrm{C}$, and supernatants were transferred to a fresh tube. Supernatants were collected 


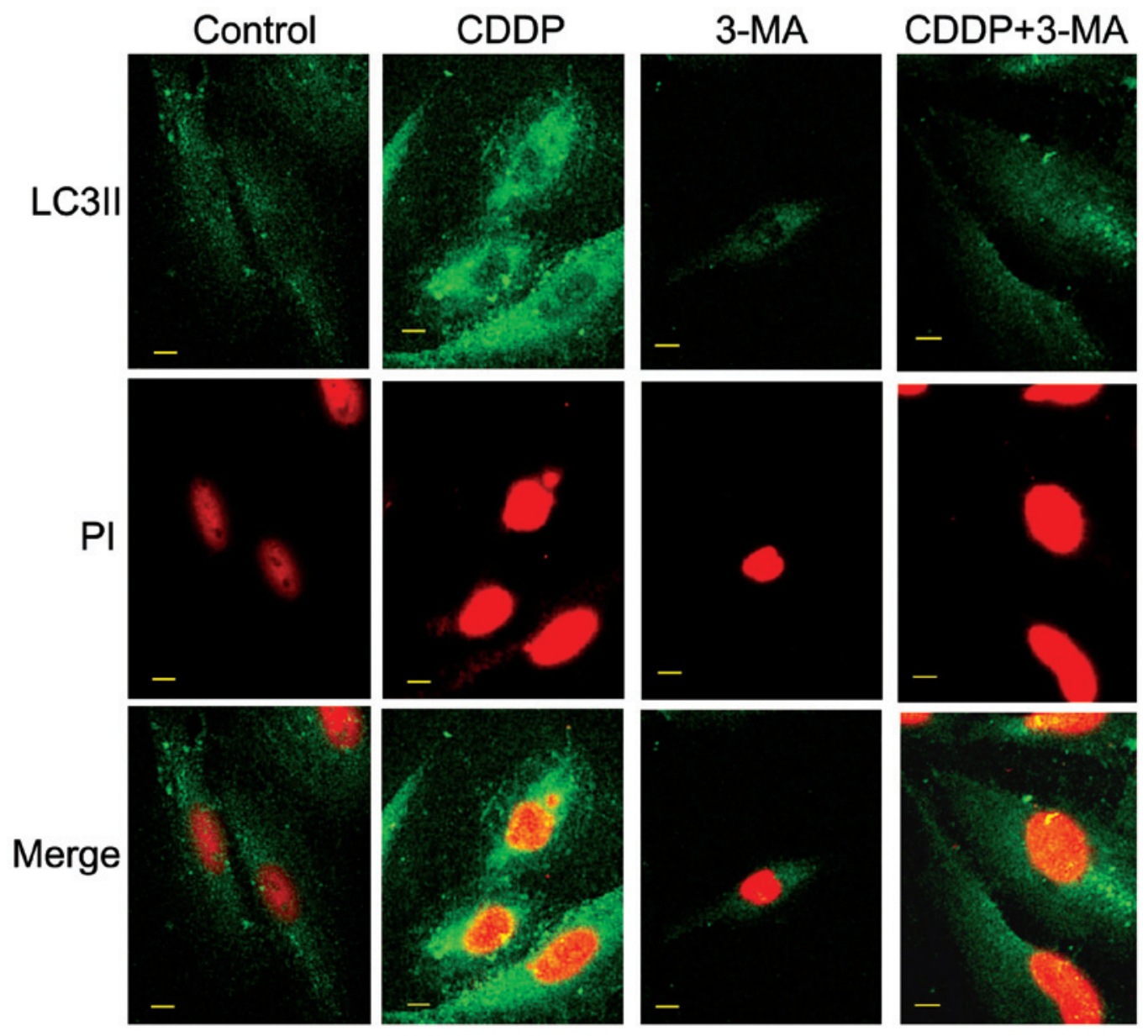

Figure 2. Representative confocal microscopy images of LC3II expression in MG63 cells. LC3II was detected using an anti-LC3II primary antibody and a fluorescein isothiocyanate-conjugated secondary antibody. The appearance of chromatin was indicated by propidium iodide staining. Green color represents localization of LC3II, which indicates autophagic activity. Red color represents localization of nuclei. Following $24 \mathrm{~h}$ of culture with no drug (control), MG63 cells demonstrated few autophagic changes. Treatment with $10 \mu \mathrm{g} / \mathrm{ml}$ CDDP significantly increased these changes. Treatment with 3-MA (6 mM) partially inhibited the expression of LC3II in MG63 cells. Co-treatment with CDDP $(10 \mu \mathrm{g} / \mathrm{ml})$ and 3-MA $(6 \mathrm{mM})$, resulted in downregulated fluorescence intensity of LC3II compared with that of cells treated with CDDP alone. Scale bar, $10 \mu \mathrm{m}$. LC3II, microtubule-associated protein light chain 3II; CDDP, cisplatin; 3-MA, 3-methyladenine; PI, propidium iodide.

as a cytosolic fraction. Protein samples $(30 \mathrm{mg})$ were electrophoresed on a $12.5 \%$ SDS gel (Clontech Laboratories, Inc.) and transferred onto a Hybond enhanced chemiluminescence (ECL) nitrocellulose membrane (Amresco, LLC, Solon, OH, USA). Membranes were blocked by incubation with $4 \%$ skim milk in PBS with Tween (PBST; Clontech Laboratories, Inc.) at $4^{\circ} \mathrm{C}$ overnight. Following rinsing, membranes were incubated with rabbit anti-human polyclonal anti-LC3II and rabbit anti-human monoclonal anti-caspase-3 (\#N791; Amresco) antibodies (1:1,000 dilution), respectively, overnight at $4^{\circ} \mathrm{C}$. Following five washes with PBST, membranes were incubated with the secondary antibody (horseradish peroxidase-conjugated anti-rabbit IgG antibody; Amresco) for $1 \mathrm{~h}$ at room temperature. Following rewashing with PBST (5 times), the membranes were analyzed using an ECL Western Blotting Substrate Kit (Amresco). X-ray films were scanned on a flat-bed scanner, and western blotting results were quantified using Photoshop Image Analysis software CS3 (Adobe Systems, Inc., San Jose, CA, USA).

Statistical analysis. SPSS 10.0 package (SPSS, Inc., Chicago, IL, USA) was utilized for statistical analysis. Data are expressed

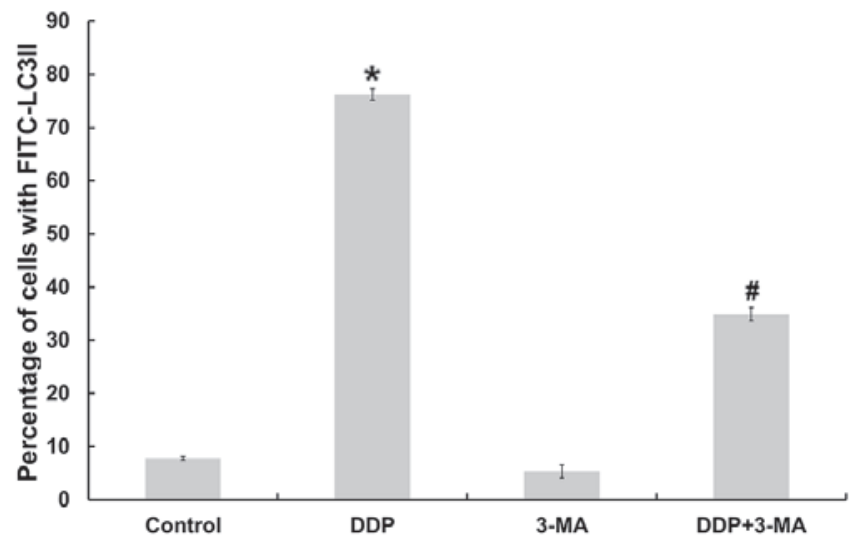

Figure 3. Quantification of autophagy in MG63 cells treated with certain drugs. Cells were collected at the indicated times and evaluated by flow cytometry. Results are presented as the mean \pm standard error with fluorescein isothiocyanate-LC3II positive dots for combined data from four separate experiments. MG63 cells produced significantly more LC3II following CDDP treatment $\left({ }^{*} \mathrm{P}<0.05\right)$. By contrast, following treatment with CDDP + 3-MA, the fluorescence intensity of LC3II was downregulated compared with cells treated with CDDP alone $\left({ }^{\#} \mathrm{P}<0.05\right)$. There was no significant difference between the untreated control and 3-MA groups $(\mathrm{P}>0.05)$. LC3II, microtubule-associated protein light chain 3II; CDDP, cisplatin; 3-MA, 3-methyladenine; FITC, fluorescein isothiocyanate. 
A
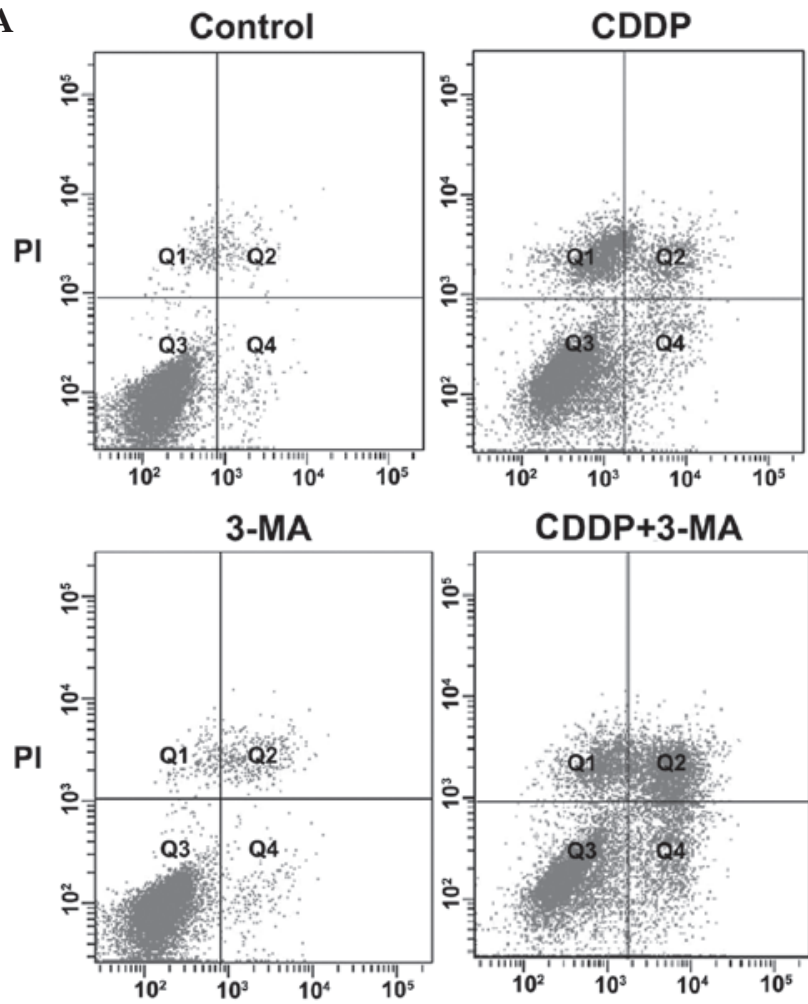

Annexin V-FITC

B

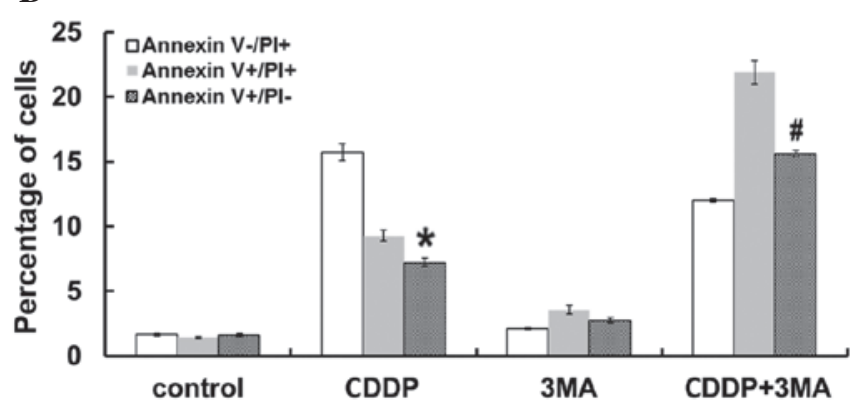

Figure 4. Flow cytometric analysis of MG63 cell apoptosis. (A) Representative plots of Annexin V vs. PI fluorescence for control cells and cells treated with CDDP, 3-MA and CDDP + 3-MA for $24 \mathrm{~h}$. Q1, Annexin $\mathrm{V}^{-} / \mathrm{PI}^{+}$, damaged cells; Q2, Annexin $\mathrm{V}^{+} / \mathrm{PI}^{+}$, necrotic cells; Q3, Annexin $\mathrm{V}^{-} / \mathrm{PI}^{-}$, viable cells; and $\mathrm{Q} 4$, Annexin $\mathrm{V}^{+} / \mathrm{PI}^{-}$, apoptotic cells. (B) Percentage of cells labeled with Annexin $\mathrm{V}^{+} / \mathrm{PI}^{-}$(apoptotic cells), Annexin $\mathrm{V}^{-} / \mathrm{PI}^{+}$(damaged cells) and Annexin $\mathrm{V}^{+} / \mathrm{PI}^{+}$(necrotic cells) following $24 \mathrm{~h}$ of treatment. Data are presented as the mean \pm standard error. CDDP significantly increased the percentage of Annexin $\mathrm{V}^{+} / \mathrm{PI}^{-}$(apoptotic cells) compared with that of control cells $\left({ }^{*} \mathrm{P}<0.05\right) .3-\mathrm{MA}+\mathrm{CDDP}$ co-treatment significantly increased the percentage of Annexin $\mathrm{V}^{+} / \mathrm{PI}^{-}$(apoptotic cells) compared with that of cells treated with CDDP alone $\left({ }^{*} \mathrm{P}<0.05\right)$. There was no significant difference between the untreated control and 3-MA groups ( $\mathrm{P}>0.05)$. PI, propidium iodide; $\mathrm{CDDP}$, cisplatin; 3-MA, 3-methyladenine; FITC, fluorescein isothiocyanate.

as the mean \pm standard error. Single-factor analysis of variance was performed for each treatment group. $\mathrm{P}<0.05$ was considered to indicate a statistically significant difference. Experiments were repeated at least three times for each condition.

\section{Results}

Inhibition of autophagy enhances CDDP-induced cell death. A previous study revealed that CDDP promotes apoptosis in OC cells (12). To determine the cytotoxicity of CDDP and
A

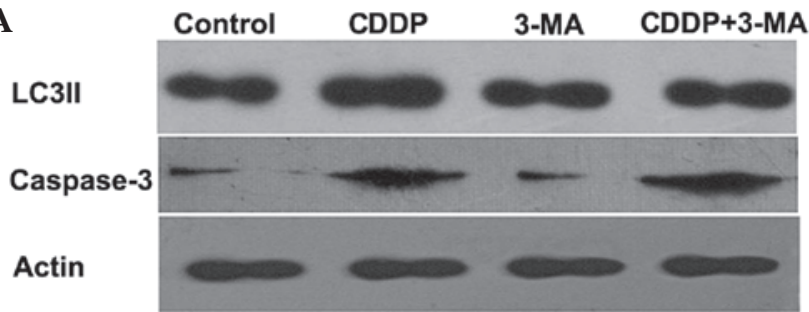

B

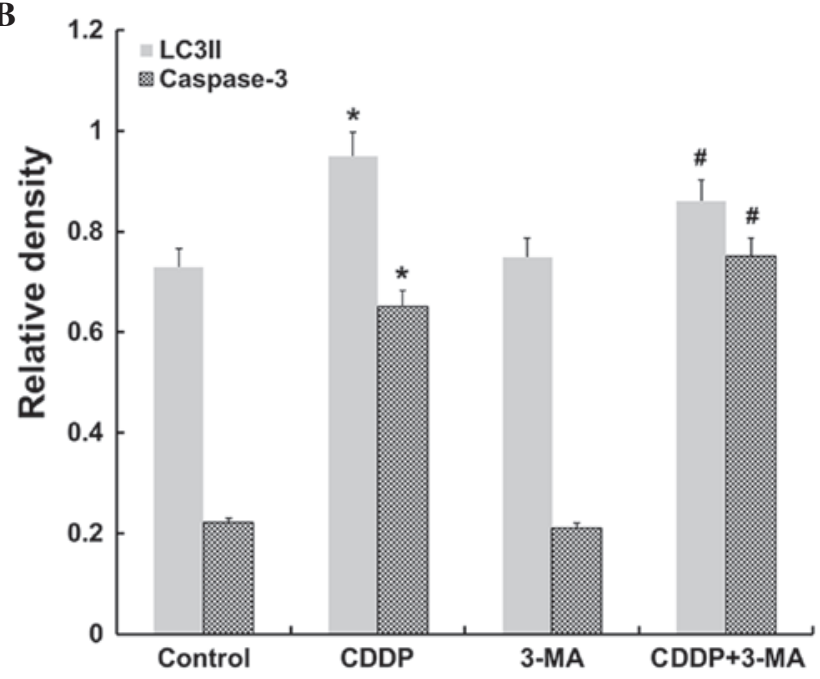

Figure 5. LC3II and caspase-3 expression in MG63 cells. (A) Western blot analysis of LC3II and caspase-3 expression in the cell populations. (B) The expression of LC3II and caspase-3 proteins was increased following CDDP treatment $\left({ }^{*} \mathrm{P}<0.05\right)$, while combined CDDP +3 -MA treatment downregulated the expression of LC3II and upregulated the expression of caspase-3 $\left({ }^{*} \mathrm{P}<0.05\right)$. There was no significant difference between the control and 3-MA groups $(\mathrm{P}>0.05)$. Data are expressed as the mean \pm standard error. LC3II, microtubule-associated protein light chain 3II; CDDP, cisplatin; 3-MA, 3-methyladenine.

3-MA on MG63 cells, the present study investigated the effects of these drugs on cell proliferation using an MTT assay. As illustrated in Fig. 1, CDDP significantly inhibited the growth of MG63 cells $(\mathrm{P}<0.05)$. The 3-MA-treated cells also demonstrated a slight change in the inhibition ratio of cell proliferation. To determine if autophagy was involved in CDDP-induced cell death, the present study examined whether 3-MA was able to inhibit CDDP-induced cell death. The cell proliferation inhibition ratio in the CDDP + 3-MA group was significantly increased compared with that of the CDDP group $(\mathrm{P}<0.05)$. These results suggest that 3-MA treatment increases the sensitivity of MG63 cells to CDDP-induced cell death.

CDDP treatment induces autophagy in MG63 cells. Autophagy is an alternative form of cell death to apoptosis, characterized by the generation of autophagic vesicles, and the degradation of cytoplasmic components and organelles (13). LC3II, a novel autophagosome marker, was utilized in the present study (14). In order to confirm the role of CDDP-induced autophagy, the present study investigated the expression of LC3II in MG63 cells using confocal microscopy. As illustrated in Fig. 2, the fluorescence intensity of LC3II was upregulated following CDDP treatment, indicating that CDDP may induce autophagy. Inhibitor of 
autophagy 3-MA partially obstructed the expression of LC3II in MG63 cells.

In order to further determine whether CDDP was capable of inducing autophagy, the present study monitored the expression profile of LC3II in cultured MG63 cells using flow cytometry. As illustrated in Fig. 3, MG63 cells produced significantly more LC3II following CDDP treatment compared with the control group cells $(\mathrm{P}<0.05)$. By contrast, following co-treatment with CDDP and 3-MA, the fluorescence intensity of LC3II was downregulated compared with that of cells treated with CDDP alone $(\mathrm{P}<0.05)$.

Inhibition of autophagy increases CDDP-induced apoptosis of MG63 cells. To investigate the association between autophagy and apoptosis in MG63 cells treated with CDDP, MG63 cells were co-treated with CDDP and 3-MA and the degree of apoptosis was assessed. The percentage of cells undergoing apoptosis was quantified using flow cytometry. Apoptotic (Annexin $\mathrm{V}^{+} / \mathrm{PI}^{-}$) and necrotic (Annexin $\mathrm{V}^{+} / \mathrm{PI}^{+}$) cells were distinguished on the basis of double-labeling for Annexin V-FITC and PI (Fig. 4A). As indicated in Fig. 4B, untreated control MG63 cells demonstrated slight fluorescent staining. By contrast, treatment with CDDP alone for $24 \mathrm{~h}$ induced a significant increase in the levels of apoptosis in MG63 cells compared with control cells ( $\mathrm{P}<0.05)$, and CDDP and 3-MA co-treatment significantly increased the percentage of apoptotic MG63 cells compared with that of cells treated with CDDP alone $(\mathrm{P}<0.05)$. Following $24 \mathrm{~h}$ of incubation, there was no significant increase in the number of necrotic (Annexin $\mathrm{V}^{+} / \mathrm{PI}^{+}$) and damaged (Annexin $\mathrm{V}^{-} / \mathrm{PI}^{+}$) cells between the untreated control group and the 3-MA-treated group. Thus, the results of the present study demonstrated that inhibition of autophagy by 3-MA significantly increased the apoptotic effects of CDDP in MG63 cells.

Differential effects of CDDP and 3-MA may be due to the expression of LC3II and caspase-3 in MG63 cells. The present study investigated the protein expression of LC3II and caspase-3 in MG63 cells. As illustrated in Fig. 5, western blot analyses revealed that the expression levels of LC3II and caspase-3 proteins were increased in MG63 cells following CDDP treatment. Furthermore, reduced levels of LC3II protein and increased levels of caspase- 3 protein were detected in the CDDP + 3-MA co-treatment group, compared with those of the CDDP-treated group $(\mathrm{P}<0.05)$. The results of the present study suggest that the differential effects of CDDP and 3-MA on MG63 cells may be due to the expression of proapoptotic and autophagic proteins.

\section{Discussion}

The results of the present study revealed that autophagy protected MG63 human OC cells from CDDP-induced apoptosis, and inhibition of autophagy mediated by 3-MA enhanced the sensitivity of MG63 cells to the apoptosis inducer CDDP. CDDP is an alkylating agent that reacts with DNA and cellular proteins, and the magnitude of cell death is well correlated with tumor response to CDDP (15). The molecular mechanisms through which CDDP induces apoptosis are DNA crosslinking, inhibition of DNA replication and RNA transcription (16). In the present study, it was demonstrated that CDDP specifically induced apoptosis via caspase-3 activation in MG63 cells.

Although a number of researchers have hypothesized potential mechanisms, the signaling pathways of the target molecules of CDDP that lead to cell death have not yet been elucidated (17). Previous studies have revealed that numerous chemotherapeutic agents are capable of inducing autophagic cell death. The alkylating agent temozolomide is capable of killing malignant glioma cells via the process of autophagic cell death (18). Comparably, resveratrol is capable of inducing autophagic cell death in ovarian cancer cells (19).

Autophagy is characterized by the appearance of abundant cytoplasmic autophagic vacuoles, and an increase in the size of the endoplasmic reticulum and Golgi apparatus (20). The LC3II protein, located in the autophagosomal membrane, may be used as a general marker for the autophagic membrane (21). 3-MA is an inhibitor of the class III phosphatidylinositol 3-kinases and is known to be involved in the initial phase of autophagy (22). A previous study investigating 3-MA demonstrated that it was capable of increasing the sensitivity of HT-29 colon cancer cells to apoptosis induced by a cyclooxygenase inhibitor, sulindac sulfide (23). In the present study, 3-MA was used in combination with CDDP, and the results revealed that the sensitivity to chemotherapy may be increased by the downregulation of autophagy. The findings of the aforementioned studies suggest that autophagy may inhibit apoptosis by sequestrating mitochondrial death-promoting factors, for example cytochrome $c$ (24). However, the mechanism of autophagic activity, which promotes the protection of cells from apoptosis remains to be elucidated.

Recent advances in our understanding of the molecular mechanisms underlying anticancer therapy-induced autophagy and apoptosis, have provided significant information for studying tumor responses, in terms of the signal transduction pathways of cell death (25). This understanding potentially facilitates the design of targeted therapies for the promotion of cancer cell sensitivity to anticancer treatments.

\section{References}

1. Desandes E: Survival from adolescent cancer. Cancer Treat Rev 33: 609-615, 2007.

2. Biermann JS, Adkins D, Benjamin R, Brigman B, Chow W, Conrad EU III, Frassica D, Frassica FJ, George S, Healey JH, et al; National Comprehensive Cancer Network: Bone cancer. J Natl Compr Canc Netw 5: 420-437, 2007.

3. Sakamoto A and Iwamoto Y: Current status and perspectives regarding the treatment of osteo-sarcoma: Chemotherapy. Rev Recent Clin Trials 3: 228-231, 2008.

4. Yamamoto $\mathrm{N}$ and Tsuchiya $\mathrm{H}$ : Chemotherapy for osteosarcoma where does it come from? What is it? Where is it going? Expert Opin Pharmacother 14: 2183-2193, 2013.

5. Shen HM and Tergaonkar V: NFkappaB signaling in carcinogenesis and as a potential molecular target for cancer therapy. Apoptosis 14: 348-363, 2009.

6. Monastyrska I and Klionsky DJ: Autophagy in organelle homeostasis: Peroxisome turnover. Mol Aspects Med 27: 483-494, 2006.

7. Shintani T and Klionsky DJ: Autophagy in health and disease: A double-edged sword. Science 306: 990-995, 2004.

8. Gewirtz DA: Autophagy as a mechanism of radiation sensitization in breast tumor cells. Autophagy 3: 249-250, 2007.

9. Lemasters JJ, Nieminen AL, Qian T, Trost LC, Elmore SP, Nishimura Y, Crowe RA, Cascio WE, Bradham CA, Brenner DA, et al: The mitochondrial permeability transition in cell death: A common mechanism in necrosis, apoptosis and autophagy. Biochim Biophys Acta 1366: 177-196, 1998. 
10. Zhang Z, Shao Z, Xiong L, Che B, Deng C and Xu W: Expression of Beclin1 in osteosarcoma and the effects of down-regulation of autophagy on the chemotherapeutic sensitivity. J Huazhong Univ Sci Technolog Med Sci 29: 737-740, 2009.

11. Wu J, Dang Y, Su W, Liu C, Ma H, Shan Y, Pei Y, Wan B, Guo J and $\mathrm{Yu} \mathrm{L}$ : Molecular cloning and characterization of rat LC3A and LC3B - two novel markers of autophagosome. Biochem Biophys Res Commun 339: 437-442, 2006.

12. Seki K, Yoshikawa H, Shiiki K, Hamada Y, Akamatsu N and Tasaka K: Cisplatin (CDDP) specifically induces apoptosis via sequential activation of caspase-8, -3 and -6 in osteosarcoma. Cancer Chemother Pharmacol 45: 199-206, 2000.

13. Suzuki K, Kubota Y, Sekito T and Ohsumi Y: Hierarchy of Atg proteins in pre-autophagosomal structure organization. Genes Cells 12: 209-218, 2007.

14. Halpain S and Dehmelt L: The MAP1 family of microtubule-associated proteins. Genome Biol 7: 224, 2006.

15. Nooter K and Stoter G: Molecular mechanisms of multidrug resistance in cancer chemotherapy. Pathol Res Pract 192: 768-780, 1996.

16. Ogawa M: Anticancer drugs and pharmacologic actions. Nihon Rinsho 55: 1017-1023, 1997 (In Japanese).

17. Park YP, Kim KD, Kang SH, Yoon Y, Park JW, Kim JW and Lee HG: Human telomerase reverse transcriptase (hTERT): A target molecule for the treatment of cisplatin-resistant tumors. Korean J Lab Med 28: 430-437, 2008.
18. Kanzawa T, Germano IM,Komata T, Ito H, Kondo Y and Kondo S: Role of autophagy in temozolomide-induced cytotoxicity for malignant glioma cells. Cell Death Differ 11: 448-457, 2004

19. Opipari AW Jr, Tan L, Boitano AE, Sorenson DR, Aurora A and Liu JR: Resveratrol-induced autophagocytosis in ovarian cancer cells. Cancer Res 64: 696-703, 2004.

20. Xie Z and Klionsky DJ: Autophagosome formation: Core machinery and adaptations. Nat Cell Biol 9: 1102-1109, 2007.

21. Tanida I, Ueno T and Kominami E: LC3 conjugation system in mammalian autophagy. Int $\mathrm{J}$ Biochem Cell Biol 36: 2503-2518, 2004.

22. Yang YP, Liang ZQ, Gu ZL and Qin ZH: Molecular mechanism and regulation of autophagy. Acta Pharmacol Sin 26: 1421-1434, 2005.

23. Bauvy C, Gane P, Arico S, Codogno P and Ogier-Denis E: Autophagy delays sulindac sulfide-induced apoptosis in the human intestinal colon cancer cell line HT-29. Exp Cell Res 268: 139-149, 2001.

24. Herman-Antosiewicz A, Johnson DE and Singh SV: Sulforaphane causes autophagy to inhibit release of cytochrome $c$ and apoptosis in human prostate cancer cells. Cancer Res 66: 5828-5835, 2006.

25. Sui X, Kong N, Ye L, Han W, Zhou J, Zhang Q, He C and Pan H: p38 and JNK MAPK pathways control the balance of apoptosis and autophagy in response to chemotherapeutic agents. Cancer Lett 344: 174-179, 2014 